\title{
A systematic optimization procedure of antenna miniaturization for efficient wireless energy transfer
}

\author{
Mohamad Harris Misran', Sharul Kamal Abdul Rahim², Maizatul Alice Meor Said³, \\ Mohd Azlishah Othman ${ }^{4}$ \\ ${ }^{1,3,4}$ Faculty of Electronics and Computer Engineering, Universiti Teknikal Malaysia Melaka, Malaysia \\ ${ }^{2}$ Wireless Communication Centre (WCC), Fakulti Kejuruteraan Elektrik, Universiti Teknologi Malaysia, Malaysia
}

\section{Article Info}

Article history:

Received Dec 10, 2018

Revised Mar 28, 2019

Accepted Apr 8, 2019

\section{Keywords:}

Near-field communication

Wireless energy transfer

Wireless transfer efficiency

\begin{abstract}
This paper presents a systematic optimization procedure to determine the reduced antenna size aimed at obtaining the best efficiency or at least equal performance with the initial large antenna design in a wireless energy transfer (WET) system. A low-cost, square-shaped planar loop antenna designed on each side of FR4 substrate is used as both the miniature transmitter and receiver antennas operating at $13.56 \mathrm{MHz}$ for the near-field communication (NFC) band. The effect of distance and antenna size on the link parameters such as inductance, resistance and mutual coupling is studied, prior to the study of their effects on WTE. The accuracy of the procedure is cross-validated using two methods; analytically and using full wave simulations. The simulation then is verified using lab measurement setup at real scene environment. Trends of the resulting curves using both methods indicated good agreements, and optimal miniature antenna for the best wireless transfer efficiency (WTE) is able to be quickly determined. A miniature antenna is able to achieve $4 \%$ wireless transfer efficiency improvement with $47 \%$ antenna size reduction. Such method can be applied to efficiently estimate a low-cost WTE system setup, besides enabling the integration of self-tuning or reconfigurability features in such systems for a known initial antenna size to mitigate changes to its operating distance.
\end{abstract}

Copyright () 2019 Institute of Advanced Engineering and Science. All rights reserved.

\section{Corresponding Author:}

Mohamad Harris Misran,

Centre for Telecommunication Research and Innovation (CeTRI),

Fakulti Kejuruteraan Elektronik \& Kejuruteraan Komputer (FKEKK),

Universiti Teknikal Malaysia Melaka (UTeM),

Jalan Hang Tuah Jaya, 76100 Durian Tunggal, Melaka, Malaysia.

Email: harris@utem.edu.my

\section{INTRODUCTION}

The power management aspect is one of the most critical issues in improving the attractiveness of mobile devices of today. Besides ideally required to be capable of powering these devices for longer durations, another interesting feature preferred by consumers is the wireless connectivity to accessories such as earphone and chargers. Such cordless recharging system is convenient and wireless energy transfer (WET) systems can provide this solution. In WET, one of the most challenging issues is maximizing its wireless transfer efficiency (WTE). The WTE in all WET systems are dependent on parameters such as frequency, size, distance or bandwidth. WET has been researched intensively at several operating frequencies, ranging from the high $912 \mathrm{MHz}$ [1] to lower frequencies such as $13.56 \mathrm{MHz}$ and $115.6 \mathrm{kHz}$ [2-3]. Several popular methods in enabling WET include techniques such as inductive coupling [4], resonant coupling [5], capacitive coupling [6] and electromagnetic coupling [7]. For near field WET systems, inductive coupling is widely investigated compared to other methods such as capacitive coupling due to safety concerns. Various materials have also been implemented in WET systems for different purposes, such as polydimethylsiloxane 
(PDMS) [8], Kapton polyimide [9], NiZn based substrates [10] and glass reinforced epoxy laminated sheets (such as FR4) [11]. Besides that, several methods to improve the efficiency of WET systems have been investigated. These include the use of loaded capacitance [12], antiparallel loop technique [13], higher order coil system [3] and the application of metamaterials [14].

The most challenges to make the antenna smaller and mobile are about maintaining WTE performance. Smaller antenna will ensure the mobility of the devices. Miniaturization of antenna has one significant draw back, which is antenna performance reduction. Small size antenna will has low mutual inductance, $m$ and this will result low WTE of the system. One of the methods to overcome this problem is by introducing parallel planar loop antenna (PPLA). Using this technique, two single square loop antenna is designed on both side of the antenna to increase the mutual inductance of the system. Even though the resistance of the antenna is increased due to longer total patch line, this can be solved by calculating the optimal width size of the microstrip line to reduce the total AC resistance, $R_{a c}$ of the antenna.

This paper proposes a systematic procedure in determining the optimal miniaturization of antenna and maintaining the efficiency of a WET system. This procedure uses realistic distance, a simple square loop antenna topology made using low-cost material, practical antenna sizes, and link operation in the NFC frequency band to facilitate this procedure's usage for other researchers working in the field. Moreover, the investigated width of the microstrip line is rounded to the $0.5 \mathrm{~mm}$ to ensure simplicity in design and analysis. This paper is organized as follows. The antenna topology and material will be first presented, followed by the proposed WTE system. The mathematical background in determining the critical parameters of the link is presented prior to the calculation and full-wave simulation results. The result then is validated by lab measurement setup for real environment scene. Finally, the optimal antenna design which produced most efficient for the WTE will be determined.

\section{RESEARCH METHOD}

Explaining research chronological, including research design, research procedure (in the form of algorithms, Pseudocode or other), how to test and data acquisition [1-3]. The description of the course of research should be supported references, so the explanation can be accepted scientifically [2, 4].

Theoretically, size of the antenna is one of the major factors that will influence the WTE. However, large antenna is not suitable for small devices and limits the mobility purpose. However, reducing the antenna size will result in low WTE. Smaller antenna leads to lower resistance, R and mutual inductance, $\mathrm{m}$ between both antennas. However, performance of the WET is directly proportional to the $\mathrm{m}$ of the system but inversely proportional to the R. This optimal size of link parameters enables WTE can be balanced using suitable matching circuits.

A single square-shaped loop antenna is chosen to optimize the available area as it complies with most smartphones as shown in Figure 1(a). This topology is chosen as the basis of the investigation in initial design size. The WET system uses identical antennas as transmitter and receiver as shown in Figure 1. Figure 1(a) shows the initial design of antenna configuration, single sided. Figure 1(b) shows the proposed reduced size of antenna that can operate without any WTE performance degradation. The investigated WET system consisting a pair of single square planar loop antennas (PLAs) can be illustrated as an equivalent circuit as shown in Figure 2.

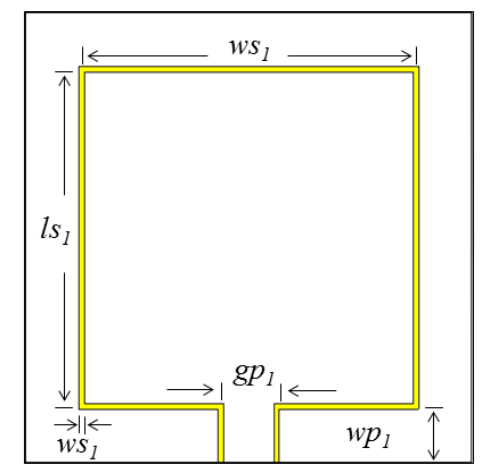

(a)

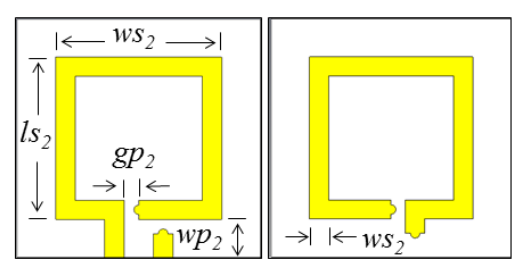

(b)

Figure 1. The (a) Initial design of PLA and (b) The reduced size design of PPLA (front and back) 


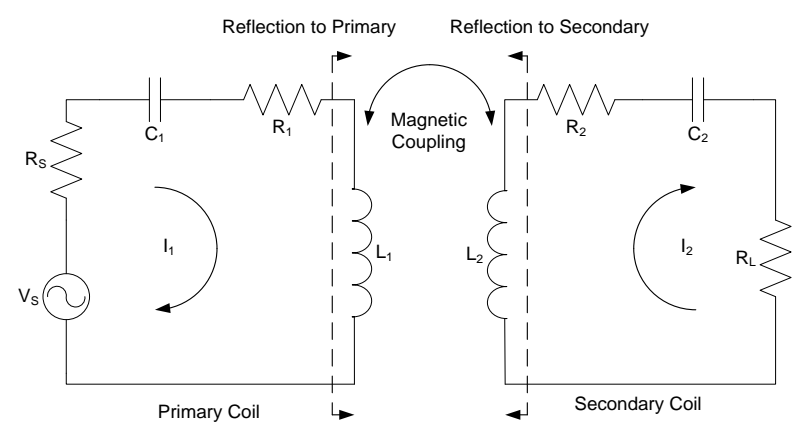

Figure 2. Equivalent circuit for antenna

The use of the loop antenna in the WET system will result in an inductive circuit rather than a capacitive for overall system. Therefore, reactance component in the system can be eliminated using an external matching circuit with two parallel capacitors to make the system resonant at $13.56 \mathrm{MHz}$ for maximum WTE.

The inductance, L of each square PLA can be calculated using [15];

$$
L=\frac{\mu n^{2} h_{\text {avg }} c_{1}}{2}\left[\ln \left(\frac{c_{2}}{\rho}\right)+c_{3} \rho+c_{4} \rho^{2}\right]
$$

where $\mu$ is the conductor permeability, which in this case is copper and $\mathrm{n}$ is number of turns of the PLA. Values of the constant $c_{1}, c_{2}, c_{3}$, and $c_{4}$ are $1.27,2.07,0.18$ and 0.13 , respectively. The fill ratio, $\rho$ and average side length of the PLAs are given by;

$$
\rho=\frac{h_{\text {out }}-h_{\text {in }}}{h_{\text {out }}+h_{\text {in }}}, \quad h_{\text {avg }}=\frac{h_{\text {out }}+h_{\text {in }}}{2}
$$

The resistance of the antenna will increase due to the skin effect at higher frequencies. Taking this into consideration, total $\mathrm{AC}$ resistance, $R_{a c}$ of the antenna at $f=13.56 \mathrm{MHz}$ can be calculated as;

$$
\begin{aligned}
& R_{d c}=\frac{\rho_{c} l}{w t_{c}}, R_{a c}=\frac{R_{d c}}{\delta}\left(\frac{t_{c}}{1-e^{-\frac{t_{c}}{\delta}}}\right) \\
& \delta=\frac{\sqrt{\rho_{c}}}{\sqrt{\pi f \mu}}, \mu=\mu_{o} \mu_{r}
\end{aligned}
$$

where $\rho_{c}$ is resistivity of copper $(1.7 \times 10-8 \mathrm{Wm}), \mu$ is the vacuum permeability and $\mu \mathrm{r}$ is the relative permeability of the copper. The conductor thickness and width are denoted as $t_{c}$ and $w$, respectively. The total length of the PLA, $l$ is determined using;

$$
l=4 h_{\text {mid }}-h_{\text {gap }}+2 h_{\text {port }}
$$

Two loop antennas close to each other will induce mutual inductance; $m$ caused by the magnetic fields, and can be calculated using [16];

$m=\frac{\mu_{o} \pi h_{t x}^{2} h_{r x}^{2}}{2\left(h_{t x}^{2}+h_{r x}^{2}+z^{2}\right)^{\frac{3}{2}}}\left(1+\frac{15}{32} \beta^{2}+\frac{315}{1024} \beta^{4}\right)\left(\frac{4}{\pi}\right)^{1+\frac{h_{\text {in }}}{h_{\text {out }}}}$ where $\beta=\frac{2 h_{t x}^{2} h_{r x}^{2}}{h_{t x}^{2}+h_{r x}^{2}+z^{2}}$

where $h_{t x}$ and $h_{r x}$ are the side lengths of the transmitter and receiver, respectively, while $z$ denotes the distance between the transmitter and receiver. The WTE of the established link is calculated as [13];

$$
\eta=\frac{k^{2} Q_{t x} Q_{r x}}{\left(1+\sqrt{1+k^{2} Q_{t x} Q_{r x}}\right)^{2}}
$$

where $Q_{t x}$ and $Q_{r x}$ are the quality factors of the transmitting and receiving antenna respectively. The coupling coefficient, $k$ between the transmitter and receiver for the system can be obtained using;

$$
k=\frac{m}{\sqrt{L_{t x} L_{r x}}}, Q_{R X}=\frac{\omega L_{r x}}{R_{r x}}, Q_{T X}=\frac{\omega L_{t x}}{R_{t x}}
$$


As comparison, a setup of the system will be simulated using CST Microwave Studio using with varying antenna parameters. Two parallel capacitors are used to match each antenna for resonance at 13.56 $\mathrm{MHz}$, as shown in Figure 3. The S-parameter is extracted from the proposed and the simulated WTE can be determined by analyzing $\left|S_{21}\right|^{2}[17]$.

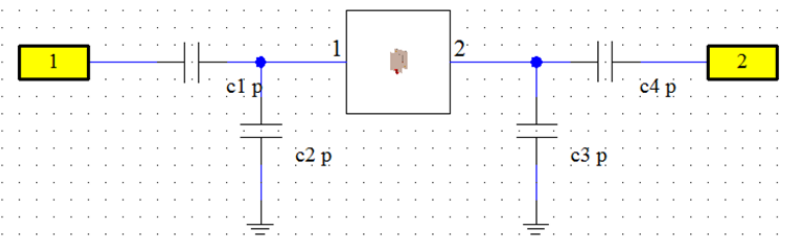

Figure 3. Matching circuit of the antenna using lump-element

\section{RESULTS AND ANALYSIS}

The antennas of both transmitter and receiver are initially sized at $80 \mathrm{~mm} \times 80 \mathrm{~mm}$ FR4 with antenna side length, ws $1=60 \mathrm{~mm}$. Microstrip line width, wl $1=0.5 \mathrm{~mm}$, port gap, pg1 $=10 \mathrm{~mm}$ and port length, $\mathrm{lp} 1=10 \mathrm{~mm}$ are being used with $40 \mathrm{~mm}$ distance between transmitter and receiver. On the other hand, the miniature antenna is designed on $50 \mathrm{~mm} \times 50 \mathrm{~mm}$ FR4, side length, ws $2=30 \mathrm{~mm}$, microstrip line width, $\mathrm{wl} 2=2 \mathrm{~mm}$, port gap, pg2 $=10 \mathrm{~mm}$ and port length, $1 \mathrm{p} 2=10 \mathrm{~mm}$ are used as parameters. All antennas are being matched using $50 \Omega$ SMA connectors at the operating frequency, $13.56 \mathrm{MHz}$. The capacitors values used for the external matching circuit are shown in Table 1.

Table 1. Capacitors value for matching circuit

\begin{tabular}{cccccc}
\hline & \multicolumn{2}{c}{ PLA } & \multicolumn{4}{c}{ PPLA } \\
$\mathrm{Cp}$ & $\mathrm{CST}$ & Actual & $\mathrm{Cp}$ & \multicolumn{2}{c}{ Value $(\mathrm{pF})$} \\
& 70.6 & 71 & $\mathrm{C}_{1}$ & 46.6 & Actual \\
\hline $\mathrm{C}_{1}$ & 448.9 & 448 & $\mathrm{C}_{2}$ & 482.4 & 482 \\
$\mathrm{C}_{2}$ & 448.5 & 448 & $\mathrm{C}_{3}$ & 481.2 & 482 \\
$\mathrm{C}_{3}$ & 70.5 & 71 & $\mathrm{C}_{4}$ & 47.3 & 47 \\
$\mathrm{C}_{4}$ & & & & &
\end{tabular}

Figure 4 shows the fabricated antenna, matched using two parallel capacitors to ensure the system resonance at NFC frequency for maximum performance. In the first stage, single square loop antennas are used for both transmitter and receiver. Then, antennas side length is reduced half from the initial design. The microstrip line width of the antennas is increased in step of $0.5 \mathrm{~mm}$ to determine the optimal size of the microstrip line width that offer higher WTE than the initial design and to ensure simplicity in design and analysis. The system is calculated theoretically, undergoing full-wave simulation and verified by lab setup measurement as shown in Figure 5. The performance of the system is monitored and analyzed.

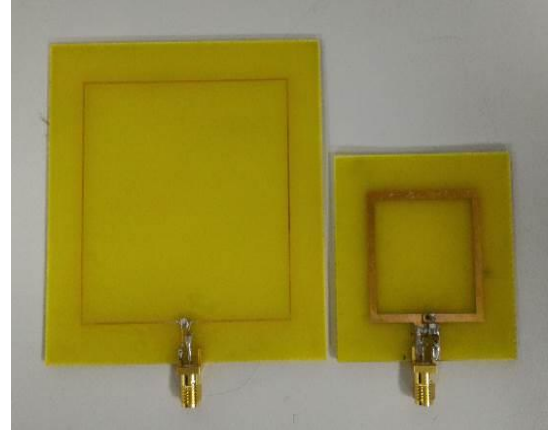

Figure 4. Fabricated antenna

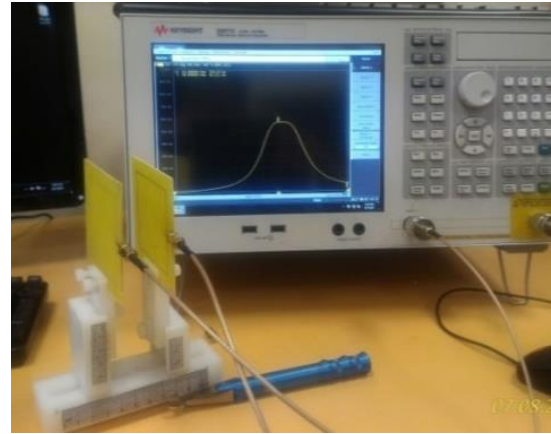

Figure 5. Lab setup for S-parameter measurement 
Generally, the total AC resistance, Rac and the mutual inductance, $m$ of the antenna is decreased when the antenna size is reduced. The reduction in $\mathrm{m}$ can be solved by adding parallel loop on the back side of the antenna. The additional loop is parallel on other side of the antenna to maximize the total area covered, hence will maximize the mutual inductance produced. The microstrip line width is widening to various size to determine the minimum size of microstrip line width for maintaining WTE of the system. When the microstrip line width is adjusting, the Q-factor, $\mathrm{m}$ and total Rac is changing concurrently.

The size reduction of the antenna contributes to less amount of the $\mathrm{m}$. This factor will affect the performance of the WET system because low m value will result to low WTE of the system. However, this problem is countered by the increment of antenna's microstrip line width and parallel loop antenna arrangement to reduce the total Rac and increase antenna's inductance, L respectively. Based on (7), $\mathrm{k}$ of the system is inversely proportional to L, hence the WTE also will be increased. Small Rac value also will produce high Q-factor, which will increase the WTE of the system.

Figure 6 shows the reduction of antenna's Rac when the microstrip line width is being increased. This is happened due to increment of cross-sectional area of the copper line. The decrement of antenna's resistance will cause the Q-factor of the antenna increase, as shown in Figure 7. Figure 8 shows that the L of the antenna also being reduced when the microstrip line width is increased, caused by the increment of fill ratio, $\rho$. Hence, $\mathrm{k}$ will increase and WTE of the system will improve as shown in Figure 9. Figure 10 shows that the WTE of the PPLA system will be higher than initial PLA design at microstrip line width equal to $2 \mathrm{~mm}$. This graph is generated by calculation in Matlab to quick determination of the optimal size of the microstrip line. Full-wave simulation is run using CST to verify the calculated value before fabrication process.

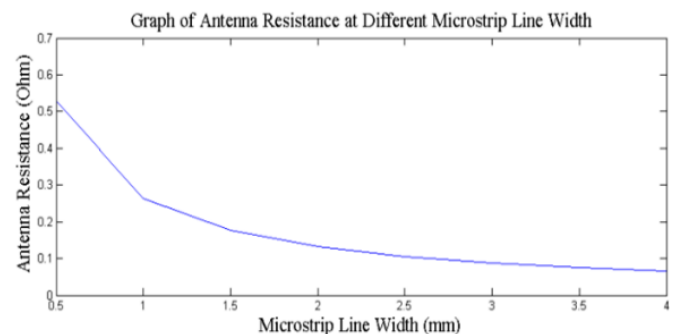

Figure 6. The antenna's resistance for various width of microstrip line

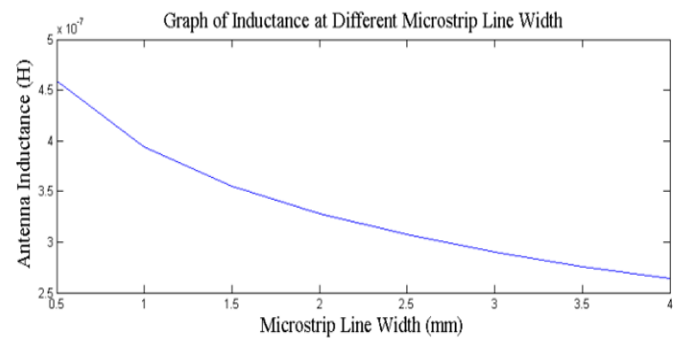

Figure 8. The antenna's inductance for various width of microstrip line

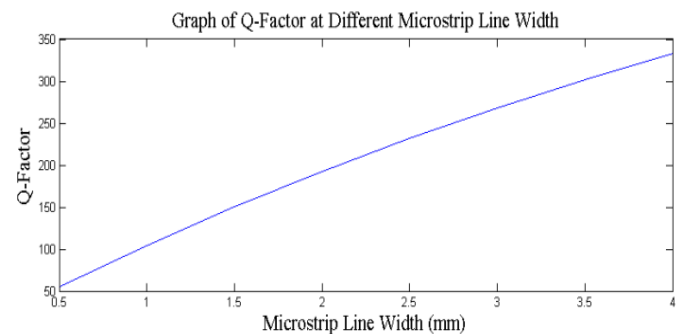

Figure 7. The $Q$-factor of the antenna for various width of microstrip line

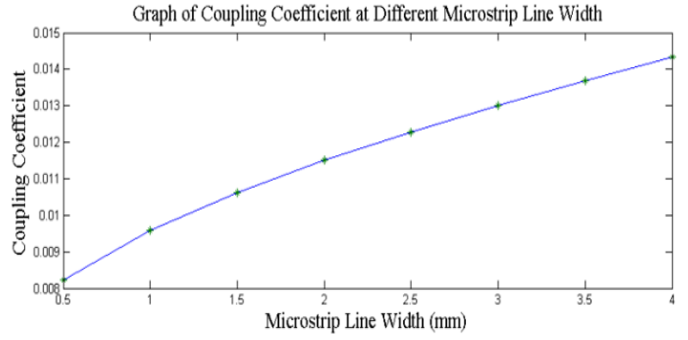

Figure 9. The coupling coefficient of the antenna, $k$ for various width of microstrip line

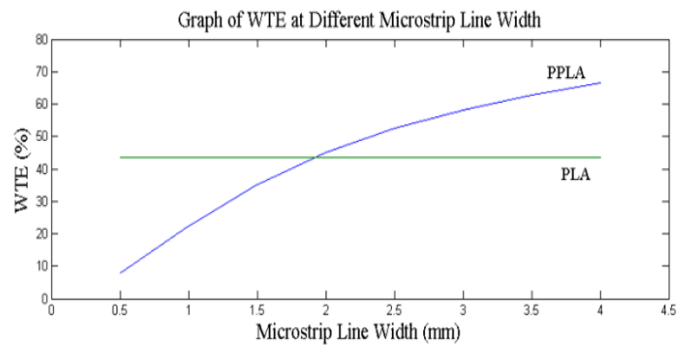

Figure 10. The WTE of the wet system for various width of microstrip line 
The simulation results of system performance for PLA and PPLA are shown is Figure 11. The trend obtained is having a good agreement with the theoretical result from Matlab. At microstrip line width is equal to 2mm, the WTE of PPLA is $6.2 \%$ higher than PLA's WTE with PPLA's size $47 \%$ smaller than PLA. From Figure 12, both results from simulation and measurement indicated good agreement, and the optimal size of PPLA is quickly obtained. The different between simulation and measurement is caused by limitation of the calculation that the formula is not able to take the substrate effect into consideration. However, the simulation form CST is completely capable to examine the substrate effect to the antenna's performance. In addition, capacitors value used for matching circuit is not really accurate because of unavailability of the component in market. Some shifting is expected and unavoidable but the different is acceptable and very insignificant.

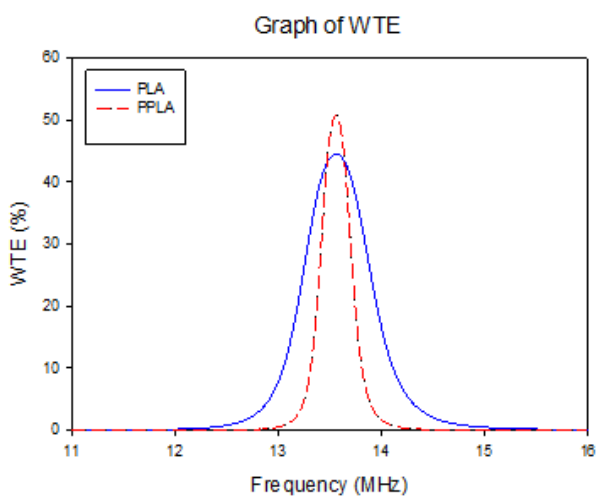

Figure 11. WTE of the PLA and PPLA from CST

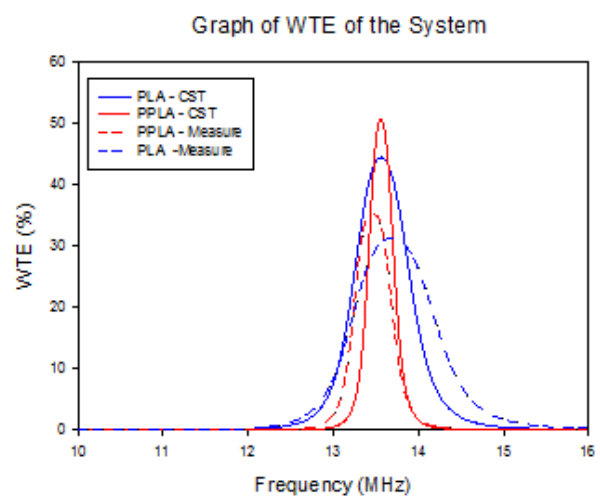

Figure 12. WTE of the system from calculation and measurement

Referring to Figure 12, the proposed PPLA offers WTE equal to $35.3 \%$ with $47 \%$ size reduction, which is $4 \%$ performance improvement comparing to fabricated PLA. The results show that the reduced antenna at almost half size can works even better than the original size of antenna version in term of WTE. Table 2 shows the summary of the PPLA improvement using double sided parallel loop technique.

Table 2. Summarization of PPLA improvement

\begin{tabular}{cccc}
\hline & PLA & PPLA & Improvement \\
\hline Size $(\mathrm{mm})$ & 61 & 32 & $47 \%$ smaller \\
WTE $(\%)$ & 31.3 & 35.3 & $4 \%$ higher \\
\hline
\end{tabular}

PPLA is matched at 40mm distance, but not limited to operate at different distances. Figure 13 shows WTE of the PPLA system at various operating distance. Even though the $m$ at closer distance is higher than $m$ at matching distance, closer distance will have splitting frequency phenomenon that will reduce the WTE of the system.

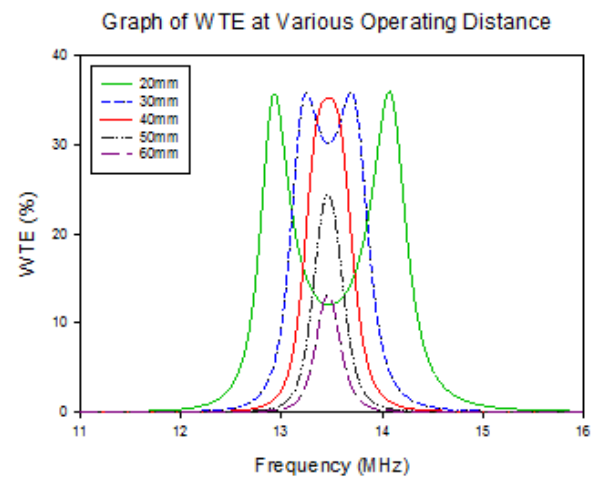

Figure 13. WTE of the PPLA at various distances 


\section{CONCLUSION}

This paper presents a systematic optimization procedure to determine the best miniature antenna's parameters for maintaining wireless transfer efficiency at $13.56 \mathrm{MHz}$. The system setup considers practical parameters such as a square-shaped PLA and PPLA fabricated using a low-cost FR4 substrate, a realistic distance between the transmitter and receiver and commonly used transmitter-receiver ratio. The properties of the WET such as resistance, inductance, $Q$-factor and mutual inductance are determined analytically and using full-wave simulations to study their contribution towards obtaining the optimal antenna's parameters value. Such method can be applied to efficiently estimate a low-cost WTE system setup, besides enabling the integration of self-tuning or reconfigurability features in such systems for a known initial antenna size to mitigate changes to its operating distance.

\section{ACKNOWLEDGEMENTS}

The authors would like to thank Universiti Teknikal Malaysia Melaka (UTeM) for supporting this research work under the grant PJP/2017/FKEKK/HI13/S01541. This research also was supported by Universiti Teknologi Malaysia.

\section{REFERENCES}

[1] J. Choo, J. Ryoo, I. Park, J. Hong, K. Park, J. Lee, "A novel multi-loop tag for near field communication in UHF band," Proceedings of Asia-Pacific Microwave Conference 2007, Dec 2007.

[2] Teck Chuan Beh, Masaki Kato, Takehiro Imura, Sehoon Oh, Yoichi Hori, "Automated impedance matching system for robust wireless power transfer via magnetic resonance coupling," IEEE Transactions on Industrial Electronics, vol. 60, no. 9, pp. 3689-3698, 2013.

[3] W. X. Zhong, C. Zhang, X. Liu, et al, "A Methodology for Making a 3-Coil Wireless Power Transfer System More Energy Efficient Than a 2-Coil Counterpart for Extended Transmission Distance," IEEE Transactions on Power Electronics, vol. 30, no. 2, pp. 933-942, 2014.

[4] R. Jegadeesan and Y.-X. Guo, "Topology selection and efficiency improvement of inductive power links," IEEE Transaction Antennas Propagation, vol. 60, no. 10, pp. 4846-4854, Oct 2012.

[5] J. S. Ho, A. J. Yeh, E. Neofytou, S. Kim, Y. Tanabe, B. Patlolla, R. E. Beygui, and A. S. Y. Poon, "Wireless power transfer to deep-tissue microimplants," Proceedings Nat. Acad. Sci. USA, vol. 111, no. 22, pp. 7974-7979, 2014.

[6] A. Sodagar and P. Amiri, "Capacitive coupling for power and data teleme- try to implantable biomedical microsystems," in Proceedings IEEE/EMBS 4th Int. Conf. Neural Eng., pp. 411-414, 2009.

[7] C. Liu,Y.-X.Guo, H. Sun, and S.Xiao, "Design and safety considerations of an implantable rectenna for far-field wireless power transfer," IEEE Transaction Antennas Propagation, vol. 62, no. 11, pp. 5798-5806, Nov. 2014.

[8] Rangarajan Jegadeesan, Sudip Nag, Kush Agarwal, Nitish V. Thakor, Yong-Xin Guo, "Enabling Wireless Powering and Telemetry for Peripheral Nerve Implants," IEEE Journal of Biomedical and Health Informatics, vol. 19, no. 3, pp. 958-970, 2015.

[9] Haider R. Khaleel, Hussain M. Al-Rizzo, Daniel G. Rucker, "Compact polyimide-based antennas for flexible displays," Journal of Display Technology, vol. 8, no. 2, pp. 91-97, 2012.

[10] Szymon Tankiewicz, Joshua Schaefer, Andrew DeHennis, "A co-planar, near field communication telemetry link for a fully-implantable glucose sensor using high permeability ferrites," 2013 IEEE SENSORS Conference, pp. 1-4, 2013.

[11] David Jugieu, Guillaume Vigneau, Mohamed Cheikh, Sebastien Kessler, Rachid Benbouhout, Alexandru Takacs, "Design and simulation of printed winding inductors for inductive wireless power charging applications," 2015 IEEE Wireless Power Transfer Conference (WPTC), pp. 1-4, 2015.

[12] W. Lee, K. OhJ. Yu, "Distance-insensitive wireless power transfer and near-field communication using a current-controlled loop with a loaded capacitance," IEEE Transaction Antennas Propagation, vol. 62, no. 2, pp. 936-940, 2014.

[13] W. Lee, W. Son, K. Oh, et al, "Contactless energy transfer systems using antiparallel resonant loops," IEEE Transactions on Industrial Electronics, vol. 60, no. 1. pp. 350-359, 2013.

[14] Gunyoung Kim, Bomson Lee, "Effects of Metamaterial Slabs Applied to Wireless Power Transfer at 13 . 56 MHz," 2015 IEEE International Symposium on Antennas and Propagation \& USNC/URSI National Radio Science Meeting, pp. 113-114, 2015.

[15] S. S. Mohan, M. del Mar Hershenson, S. P. Boyd, T. H. Lee, "Simple accurate expressions for planar spiral inductances," IEEE Journal of Solid-State Circuits, vol. 34, no. 10, pp. 1419-1424, 1999.

[16] Raju S., Rongxiang Wu, Mansun Chan, Yue C.P., "Modeling of Mutual Coupling Between Planar Inductors in Wireless Power Applications," IEEE Transactions on Power Electronics, vol. 29, no. 1, pp. 481-490, 2013.

[17] Inagaki N., "Theory of Image Impedance Matching for Inductively Coupled Power Transfer Systems," IEEE Transactions on Microwave Theory and Techniques, vol 62, no. 4, pp. 901-908, 2014. 


\section{BIOGRAPHIES OF AUTHORS}

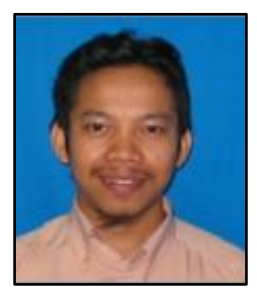

Mohamad Harris MISRAN was born in Johor, Malaysia He obtained his degree in BEng in Electronics Engineering (Telecommunication) from University of Surrey, UK in 2006 and MEng in Master of Engineering (Telecommunication) in 2008 from University of Wollongong, Ausralia. He is currently a full-time Ph.D. research student at the Wireless Communication Centre (WCC), Faculty of Electrical Engineering, Universiti Teknologi Malaysia (UTM), Johor, Malaysia.

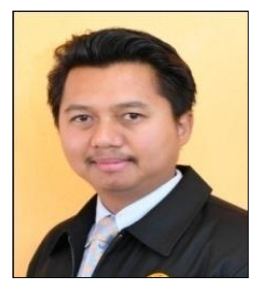

Sharul Kamal ABDUL RAHIM received his first degree from University of Tennessee, USA majoring in Electrical Engineering, graduating in 1996, M. Sc in Engineering (Communication Engineering) from Universiti Teknologi Malaysia (UTM) in 2001, and PhD. in Wireless Communication System from University of Birmingham, UK in 2007. Currently, he is an Associate Professor at Wireless Communication Centre, Faculty of Electrical Engineering, UTM. His research interest is Smart Antenna on Communication System.

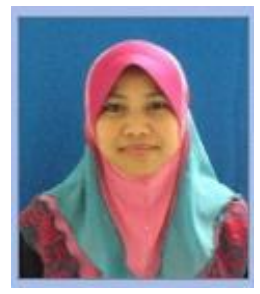

Maizatul Alice MEOR SAID received her BEng in Electronics Engineering (Telecommunication) from University of Surrey, UK in 2006. She obtained an MEng in Master of Engineering (Telecommunication) in 2009 from University of Wollongong, Ausralia. She received her Ph. D from Universiti Teknikal Malaysia Melaka (UTeM), Melaka, Malaysia in 2018.

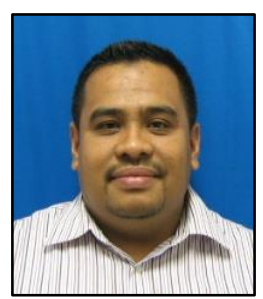

Mohd Azlishah Othman received his BEng in Electrical Engineering (Telecommunication) from Universiti Teknologi Malaysia, Malaysia, M. Sc in Computer and Communication Engineering form The University of Nottingham U.K. He received his PhD in Electronic \& Electronic Engineering also from The University of Nottingham U.K 